\title{
Induction of Forward Mutants in the pyr-3 Region of Neurospora
}

\author{
By J. L. REISSIG* \\ Institute of Genetics, University of Copenhagen, Denmark
}

(Received 7 June 1962)

\begin{abstract}
SUMMARY
Forward mutations at the pyr locus in Neurospora, and back mutations at arg, were scored by using the 'suppressor method'. This method is efficient and well suited for mutagen screening. Nitrous acid, ethylmethane sulphonate and ultraviolet radiation were effective as mutagens; 8-ethoxycaffeine yielded inconclusive results. The same pattern of forward mutations at pyr, but different frequencies of back mutations at the arg locus, were obtained with the three agents. In this Neurospora system, nitrous acid appeared to induce mutations which affected simultaneously both DNA strands.
\end{abstract}

\section{INTRODUCTION}

To facilitate studies in mutagenesis it is necessary to have screening methods, both simple and thorough, for the selection of forward and back mutants at specific loci (Westergaard, 1960). A particularly promising forward selection method is available for the pyr-3 ( pyr) locus of Neurospora. This method was described in the first paper of this series (Reissig, 1960), and will be henceforth called the suppressor method. It can be outlined as follows: uninucleate conidia (microconidia) from an arginine-dependent strain of Neurospora are plated on medium supplemented with pyrimidine, but lacking arginine. Arginine-dependence in this strain is determined by the arg gene. The arginine-independent colonies which grow on the screening plates result from any of the following events: (i) back mutation at arg; (ii) forward mutation from $p^{+} r^{+}$to $p y r$, having the pleiotropic effect of suppressing the arginine requirement while creating a requirement for pyrimidines (mosaic $p y r^{+} /$pyr colonies also grow, because the suppressor effect of pyr is dominant); (iii) forward mutation

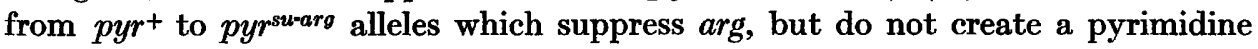
requirement. The main purpose of the present study was to test the usefulness of the suppressor method for: $(a)$ screening mutagens; $(b)$ analysing patterns of mutagen specificity; (c) determining how many of the chains in the DNA duplex are altered per mutagenic hit. It was of particular interest to test $(c)$ for nitrous acid, since Tessman (1959) and Vielmetter \& Wieder (1959) showed that nitrous acid produced single-chain mutagenic hits in bacteriophage. Contrary to those results, the data to be presented here suggest that nitrous acid produces mutations in Neurospora by simultaneously altering both chains in the DNA molecule.

The following abbreviations are used: $p y r$ for $p y r-3$; $a r g$ for arg-2.

* Present address : Laboratorio de Genética, Facultad de Ciencias Exactas y Naturales, Perú 222, Buenos Aires, Argentina. 


\section{METHODS}

Neurospora strains. Strain ED416-1 a carries the arg (Srb \& Horowitz, 1944), cot (Mitchell \& Mitchell, 1954), $p e^{m}$ and $f$ (Barratt \& Garnjobst, 1949) markers. In consequence it is arginine-dependent, colonial at temperatures above $31^{\circ}$, and microconidial. Microscopic observation by using Giemsa staining showed that $98 \%$ of the microconidia were uninucleate and $2 \%$ binucleate.

A tester strain carried the following markers: co (Mitchell \& Mitchell, 1954), arg and pyr (Reissig, 1959).

Media. The medium of Westergaard \& Mitchell (1947) with lower sugar content $(0 \cdot 2-1 \%)$ and supplemented as required was used for crosses. The solid medium of Fries (Beadle \& Tatum, 1945), supplemented as required, was used in all other cases. Hydrolysed ribonucleic acid (RNA; 0.5 g./1.) was used as pyrimidine source; L-arginine $\mathrm{HCl}$ was added to $0 \cdot 1 \mathrm{~g} . / 1$.

Screening. Microconidia were harvested from cultures of ED 416-1 a grown at $20^{\circ}$ for a fortnight on arginine-supplemented medium. This low temperature of incubation was required to obtain good microconidial viability (F. J. de Serres, personal communication; Reissig, unpublished observations). In the experiments to be reported, the viability of untreated microconidia ranged from 18 to $61 \%$, average $31 \%$.

The microconidial suspension was filtered through sintered glass (average pore diam. $50 \mu$ ) and washed twice with water by centrifugation. Suspensions containing $10^{8}$ microconidia $/ \mathrm{ml}$. were incubated at $25^{\circ}$ with agitation under the conditions indicated below.

For treatment with ultraviolet (u.v.) radiation, an aqueous suspension of microconidia was exposed to a Hanovia Germicidal Unit for various times. The u.v. dose in ergs was determined by using the dosimeter of Latarjet. For treatment with ethylmethane sulphonate, the microconidia were incubated for $11 \mathrm{hr}$. in the $\mathrm{pH} 9$ buffer of Teorell \& Stenhagen (1938) + ethylmethane sulphonate. This procedure was developed by $\mathbf{H}$. Malling (personal communication). For treatment with nitrous acid, the microconidia were incubated for $85 \mathrm{~min}$. in $0.05 \mathrm{M}$-potassium tartrate + sodium nitrite. The nitrite was added at time zero to the incubation mixture as a freshly made, sterile filtered, aqueous solution. For treatment with $p$-benzoquinone, the microconidia were incubated for $2 \mathrm{hr}$. in half strength $\mathrm{pH} 6.5$ Teorell \& Stenhagen buffer $+p$-benzoquinone. Treatment with 8-ethoxycaffeine required incubations for 5 days in liquid minimal medium (i.e. minimal medium minus agar) +8-ethoxycaffeine. In every experiment an untreated series was incubated in a similar fashion, but without the mutagen.

The screening plates contained minimal medium supplemented as follows : bottom layer (12 ml.) with hydrolysed RNA; middle layer (3 ml.) like the bottom layer, but containing the microconidial suspension; top layer $(12 \mathrm{ml}$.) with hydrolysed RNA+ L-canavanine sulphate $(0.15 \mathrm{~g} . / \mathrm{l}$. $)+\mathrm{L}$-lysine $\mathrm{HCl}(0.05 \mathrm{~g} . / \mathrm{l}$.$) . In some experi-$ ments, an aqueous microconidial suspension was substituted for the middle layer. The top layer was required to inhibit residual growth, and was added 18-25 hr. after plating the microconidia. The plates were incubated at $32^{\circ}$, but cooled to $25^{\circ}$ for some hours on the day before scoring. Scoring was done on the 8th day, unless otherwise specified. Viability was reckoned from platings on minimal medium + arginine. 
Low plating densities and premature addition of the top layer resulted in low recoveries of mutants (Reissig, 1960; and unpublished). Since some mutagenic treatments alter the value of those parameters, each experiment was run in six series, plating microconidia at densities of $2 \times 10^{7}$ and $10^{8} / \mathrm{plate}$, and adding the top layer 18, 21 and $25 \mathrm{hr}$. after plating. Only the results of the highest-yielding series are reported. Analysis of the complete results, and reconstruction experiments, indicated that recovery was essentially quantitative for treatments with low doses of mutagens, but was occasionally incomplete at higher doses.

Other procedures. For further study, mutants were picked on to slopes supplemented with arginine + pyrimidines. Filtered microconidial suspensions from each of those cultures, at concentrations of $10^{3}-10^{4}$ viable microconidia/plate were plated on three different media: arginine supplemented, pyrimidine supplemented, and unsupplemented. These plates were used for purification of the strains, preliminary classification of nutritional requirements, and classification of the clones as pure or mosaic. Further experimental details are presented in an earlier publication (Reissig, 1960).

\section{RESULTS}

\section{Dose effects of mutagens}

Figure $1 a$ shows that $u . v$. radiation, ethylmethane sulphonate and nitrous acid were effective mutagens. The curves presented relate mutant frequency among survivors to the log of the surviving fraction. They are roughly equivalent to dose dependence curves, since the log of the surviving fraction is approximately a linear function of dose (Fig. 2).

Each microconidium receives on the average one lethal hit when the survival is $37 \%$ (Lea, 1947). The probability of a scorable mutation at this survival value can be read off Fig. $1 a$. It is $2 \times 10^{-5}$ with radiation or ethylmethane sulphonate, and $10^{-5}$ with nitrous acid. Therefore, the ratios of the probability of a lethal hit to the probability of a scorable mutation are $1: 2 \times 10^{-5}$ or $1: 10^{-5}$, according to the mutagen used. Such ratios can be taken to represent the relative size and perhaps sensitivity, of the targets involved in killing (the whole genome) and in mutation (genes pyr and arg). Yet this can be at best an approximation since the mutation frequency curves for uv radiation and ethylmethane sulphonate are non-linear. Treatment with $p$-benzoquinone kills microconidia, but induces no mutations. The data are included in Fig. 1 to show that mere killing will not simulate the induction of mutants (Grigg, 1952; Kølmark \& Westergaard, 1952). Figure $1 b$ is similar to Fig. 1 $a$, except that mutant frequency was calculated per treated microconidium instead of per surviving microconidium. Net increases in the number of mutants were observed, thus ruling out selection of pre-existing mutants as a significant factor in the results presented. In other experiments, treatment with 8-ethoxycaffeine increased mutant frequency among survivors, without effecting a net increase over the spontaneous value. Survivals were (\%) 39, 17, 10, 7, 3 and 2 under the conditions used, which involved incubation for 3-5 days with concentrations of 8 -ethoxycaffeine up to saturation, at temperatures ranging from 12 to $32^{\circ}$. Controls were incubated under the same conditions, but without 8-ethoxycaffeine. 


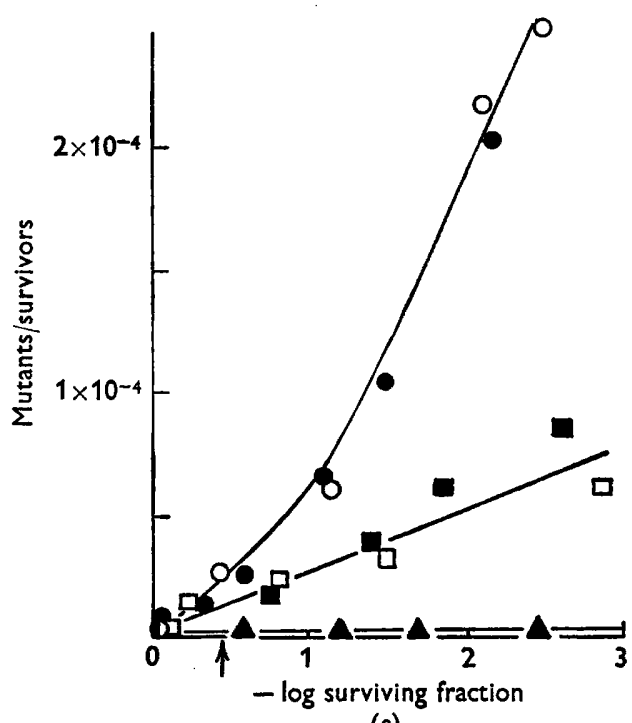

(a)

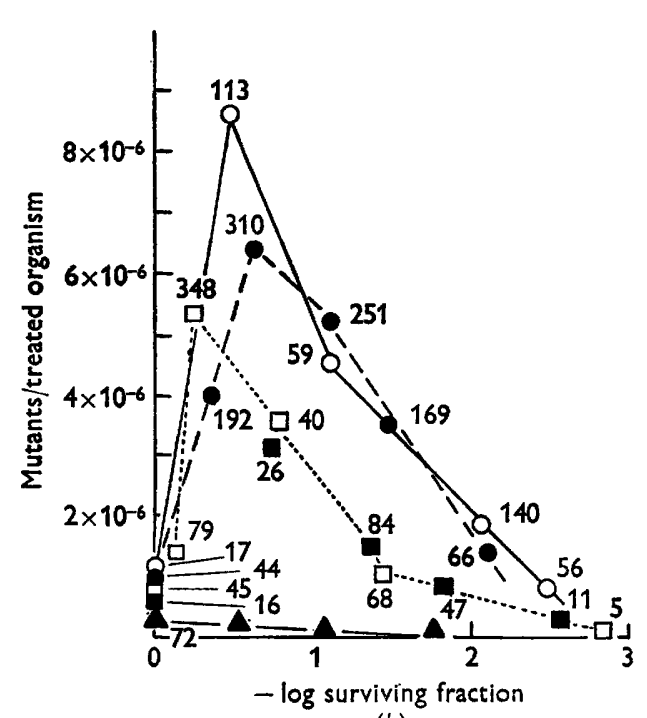

(b)

Fig. 1. Relation between mutant frequency and surviving fraction after several treatments. (a) Neurospora mutants calculated/survivor. The arrow indicates the $37 \%$ survival level. (b) Mutants calculated/microconidium viable before treatment. Treat-

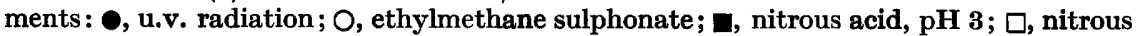
acid, $\mathrm{pH} 4 ; \boldsymbol{\Delta}, p$-benzoquinone. Actual colony counts are indicated by the numbers in the body of $(b)$.

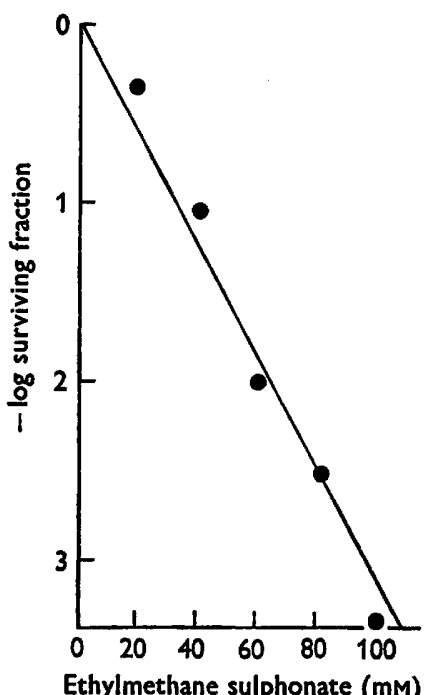

(a)

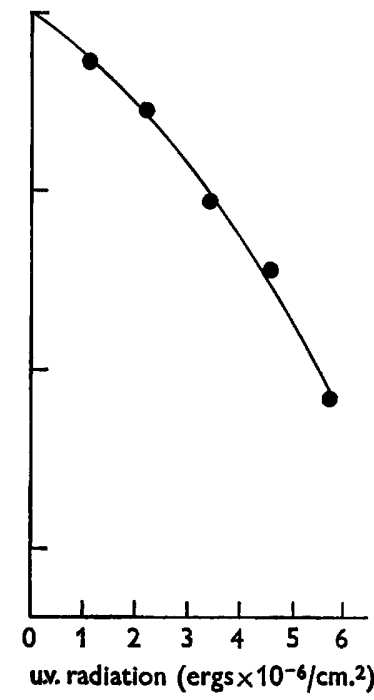

(b)

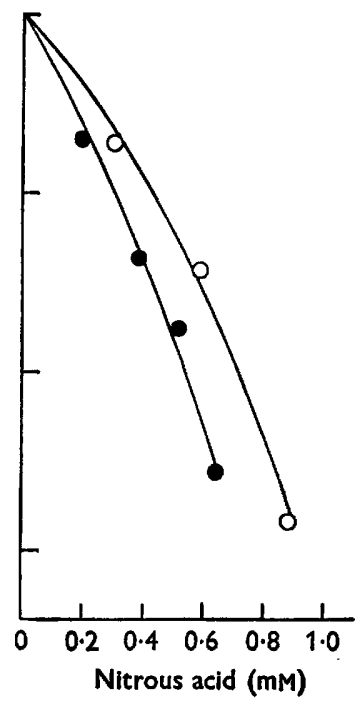

(c)

Fig. 2. Survival of Neurospora microconidia as a function of dose. Treatments: (a) ethylmethane sulphonate; (b) u.v. radiation; (c) nitrous acid. In $(c)$ two $\mathrm{pH}$ values were used: $\mathrm{pH} \mathrm{3}$; $\mathrm{O}, \mathrm{pH} \mathrm{4}$; and the plotted molarity is that of the undissociated acid, calculated from the dissociation constant of nitrous acid $\left(=5.8 \times 10^{-4}\right.$; Sneed \& Brasted, 1956), the actual pH value, and the amount of added nitrate. Data from the same experiments as in Fig. 1. 


\section{Mutagen specificity}

The suppressor method yields a variety of mutant types: pyrimidine-independent types, including back mutations at arg and mutations to pyrsura ; and pyrimidinedependent types, comprising five different complementation classes and whole range of degrees of dependence (Reissig, 1963). The question arose whether different mutagens give the same spectrum of mutant types. This is so within the pyrimidinedependent group, where the three mutagens tested yielded the same distribution of complementing types (Table 1). Furthermore, comparison of the frequency of types exhibiting residual growth among mutants induced by u.v. radiation, ethyl methane sulphonate or nitrous acid, did not reveal any mutagen-specific differences.

Table 1. Mutations in Neurospora representing the different complementation groups, classified according to origin

Methods as described in Reissig (1962)

\begin{tabular}{|c|c|c|c|c|c|}
\hline \multirow[b]{2}{*}{ Origin } & \multicolumn{5}{|c|}{ No. mutants in complementation groups } \\
\hline & Alpha & Beta & Gamma & Delta & $\begin{array}{l}\text { Non- } \\
\text { comple- } \\
\text { menting }\end{array}$ \\
\hline Spontaneous* & 4 & 1 & $\mathbf{0}$ & $\mathbf{0}$ & $\mathbf{0}$ \\
\hline U.v. radiation $†$ & 24 & 9 & 0 & 1 & 4 \\
\hline Ethylmethane sulphonate & 30 & 4 & $\mathbf{0}$ & $\mathbf{0}$ & 1 \\
\hline Nitrous acid & 97 & 24 & 3 & 0 & 2 \\
\hline Total & $\mathbf{1 5 5}$ & 38 & $\mathbf{3}$ & 1 & 7 \\
\hline
\end{tabular}

Table 2. Dependent and independent mutants of Neurospora classified according to origin

Only mutants scored on the 6th day are tabulated.

$\begin{array}{lccc}\text { Origin } & \text { Pyrimidine- } & \text { No. of mutants } & \text { Pyrimidine- } \\ \text { independent } & \begin{array}{c}\text { Pyrimidine- } \\ \text { dependent }\end{array} & \begin{array}{c}\text { independent } \\ (\%)\end{array} \\ \text { U.v. radiation } & \mathbf{8 9} & \mathbf{3 2} & \mathbf{7 4} \\ \text { Ethylmethane sulphonate } & \mathbf{4 1} & \mathbf{2 4} & \mathbf{6 3} \\ \text { Nitrous acid } & \mathbf{1 0 0} & \mathbf{5 3} & \mathbf{6 5}\end{array}$

$\boldsymbol{P}$ that the difference between u.v. mutants and the others is due to chance alone $=0 \cdot 1$.

On the other hand, relating pyrimidine-dependent to independent mutants, u.v. radiation seems to produce more of the latter than either ethylmethane sulphonate or nitrous acid (Table 2). A closer examination of this difference demands classification of the mutants into the two expected genotypes: arg $^{+}$pyr ${ }^{+}$and arg pry $\mathrm{y}^{\text {su-arg}}$, by recombination tests (Reissig, 1960). Briefly, the unknown strain is crossed to a co arg pyr tester, and the ensuing ascospores are plated on arginine medium. The few colonial recombinants obtained are then tested for arginine dependence. If the unknown parent was arg $^{+}$pyr+, most co pyr $^{+}$recombinants (35 out of 47 in the 
experiment reported below) will be arginine dependent (co $\arg \mathrm{pyr}^{+}$) because the arg-pyr distance is longer than co-arg. On the other hand, if the unknown was arg pyrsu-arg all pyrimidine-independent segregants will carry pyr $^{\text {su-arg }}$ and be thus arginine-independent. The results of such tests are reported in Table 3.

The probability of misclassification of back mutants as a result of recovering only cross-overs in the co-arg region is slight. Adequate numbers of colonial recombinants (ranging in each cross from 3 to 21 , with an average of 8 ) were tested for $\mathbf{7 7}$ of the 89 mutants classified as pyr ${ }^{\text {su-arg }}$.

It is clear the u.v. radiation induced back mutations at arg, relative to pyrsurarg events, more frequently than ethylmethane sulphonate or nitrous acid. On the basis of this result, the larger yield of prototrophs after u.v. irradiation (Table 2) can also be understood.

Table 3. Pyrimidine-independent mutants of Neurospora tested genotypically, classified according to origin

\begin{tabular}{|c|c|c|}
\hline \multirow[b]{2}{*}{ Origin } & \multicolumn{2}{|c|}{ No. mutants } \\
\hline & $\arg ^{+}$pyrt $^{+}$ & arg pyr ${ }^{8 t-a r g}$ \\
\hline Spontaneous* & $\mathbf{0}$ & $\mathbf{3}$ \\
\hline U.v. radiationt & 13 & 6 \\
\hline Ethylmethane sulphonate & $\mathbf{0}$ & 39 \\
\hline Nitrous acid & $\mathbf{5}$ & 43 \\
\hline
\end{tabular}

\section{Mechanism of action of nitrous acid}

According to current ideas, nitrous acid produces mutations by chemical modification of only one of the two chains of the DNA duplex. In the present system such one-chain hits should yield mosaic clones (mutational mosaics) containing arg pyr and arg pyr ${ }^{+}$nuclei derived respectively from the mutated and the unmutated strand. The expectation that these heterokaryons would be detectable by the usual screening method is based on the following observations : $(a)$ the suppressor effect of pyr is dominant over pyr+ (Reissig, 1958); (b) mutations decreasing pyr $^{+}$ activity to one half are readily scored by the present method (Reissig, 1963); (c) arg pyr/arg pyr+ mosaics originated by mutation of one nucleus in multinucleated macroconidia were in fact recovered (Reissig, 1960); $(d)$ when plating microconidia at high concentration, a sizable proportion of the mutant colonies analysed were arg pyr/arg pyr+ mosaics, probably as a result of contamination with the background microconidia.

The frequency of mosaics after nitrous acid treatment was determined. As a control to estimate the frequency of mosaics originating by contamination (contamination mosaics), microconidia from a pyr strain of an unusual complementation type were mixed at a concentration comparable to the level of induced mutants expected. Colonies involving the unusual pyr types were classified as control series, since almost all mosaics among them must be contamination mosaics. Colonies of other complementation types make up the test series, and mosaics in this series could be originated by mutation or by contamination. The occurrence of muta- 
tional mosaics might have been inferred if the frequency of mosaics in the test series had been significantly larger than in the control. This was not the case (Table 4).

Before disallowing the theory that nitrous acid produces mutations in this system by means of single-chain hits on the DNA duplex, we must consider the possibility that the absence of mutational mosaics is due to concurrent induction of singlechain recessive lethals which made the DNA molecule effectively single-stranded for information transfer. Now, if killing were due to single-chain recessive lethals the killing curve ought to have been typical multi-hit, with a wide shoulder and extrapolating at zero dose to $2^{\mathrm{m}}$ (on the usual log scale), where $\mathrm{m}$ is the total number of essential genes (Atwood \& Norman, 1949). This is not compatible with Fig. $2 c$. Another model postulates that each chain is essentially a single target for lethal hits which destroy the capacity of the chain to replicate. A 2-hit killing curve is expected, and this might be consistent with the data depicted in Fig. 2c. However, such a mechanism could not have prevented the detection of mosaics in the experiment reported in Table 4. There, nitrous acid killed $36 \%$ of the organisms, and the probability of a lethal hit/strand would be the square root of this, or $60 \%$. Forty $\%$ of the strands would have survived, and thus $40 \%$ of the mosaics would have been recovered. On the other hand, if killing by nitrous acid were a more complex phenomenon, combining features of the two simple models described above and possibly involving also dominant lethals and double-chain hits, then it would become impossible to decide what proportion of mosaics can be recovered.

\section{Table 4. Frequency of $\mathrm{pyr} / \mathrm{pyr}^{+}$mosaics, among mutants of Neurospora induced by nitrous acid (test series) or pre-existing (control series) \\ A $10^{5}: 1$ mixture of arg and pyr (type beta, much residual growth) Neurospora micro- conidia, treated with nitrous acid at $\mathrm{pH} 4$ and plated at a density of $10^{7}$ microconidia/ plate in a solid middle layer. Survival: $64 \%$. Frequency of induced mutants : $8 \times 10^{-6} /$ survivor. Colonies carrying the added pyr type are tabulated in the control series. Clones without $>10 \% \mathrm{pyr}^{+}$nuclei are classed as mosaics.}

No. tested mutant clones

$\begin{array}{lcc}\text { Series } & \text { Not mosaic } & \text { Mosaic } \\ \text { Test } & 99 & 4 \\ \text { Control } & \mathbf{7 3} & 6\end{array}$

\section{DISCUSSION}

The suppressor method appears very suitable for mutagen screening, being efficient and simple. Its sensitivity is only limited by the usual value of spontaneous mutants (of the order of $10^{-7}$ /live microconidium), and by recovery difficulties encountered when using highly toxic treatments (survival below $0 \cdot 1 \%$ ). Contrary to the clear-cut results obtained with u.v. radiation, ethylmethane sulphonate and nitrous acid it was not possible to decide whether or not 8-ethoxycaffeine was mutagenic. If 8-ethoxycaffeine induces mutations in the present Neurospora system, they are not frequent enough in relation to lethal events to produce a net increase in the number of mutants. Under such conditions, reconstruction experiments would be required to rule out selection as the cause for the increase in mutant 
frequency. Selective effects were particularly suspect in the present experiments because very long incubation times in minimal medium were demanded by the low solubility of 8-ethoxycaffeine. On the whole, the results obtained with this system are not unlike those reported for Ophiostoma by Zetterberg (1960), who claimed a slight mutagenic effect for 8-ethoxycaffeine on the basis of the results of reconstruction experiments.

The curves of the present work which relate mutant frequency/survivor to lethal hits ( - log of surviving fraction) after treatment with u.v. radiation or ethylmethane sulphonate, depart from the linearity which simple theory predicts. They are, however, typical of the dose curves obtained for u.v.-induced prototrophy in Neurospora (Giles, 1951) and bacteria. A plausible explanation for this effect was furnished by Witkin (1959) who worked with a bacterial system.

The suppressor method, being essentially a forward-mutation system, is probably more reliable than the usual back-mutation method for the screening of potential mutagens. Forward mutation is the summation of events at a considerable number of mutons and the specificities of their reaction average out. Back mutation, on the other hand, involves a restricted number of mutons being altered in specific ways; therefore different alleles respond specifically to different mutagens. This question was reviewed by Westergaard (1960). The contrasting behaviour of forward and back mutation is again brought out by the data here presented. The relative frequencies of $p y r-\alpha, p y r-\beta, p y r^{s u-a r g}$ mutations and lethal events were the same whether uv radiation or ethylmethane sulphonate were used as mutagens; yet $u . v$. radiation is far more effective than ethylmethane sulphonate as an inducer of back mutations at arg relative to the above-mentioned events. The obvious interpretation is that back mutation of the arg allele is mutagen specific, while forward mutation at pyr is not.

The results obtained with nitrous acid strongly suggest that this compound does not induce mosaics in Neurospora. The possibility that mosaics occur, but are not detectable, was examined; no evidence in its favour was found. Further proof would need to come from reconstruction experiments with microconidia known to be mosaic for both DNA chains, but this material is not available. Thus, on the basis of the available evidence, the absence of mosaics contradicts the hypothesis that nitrous acid produces mutations by deaminations which affect only one DNA chain/hit. Geiduschek (1961) showed that in vitro treatment of DNA by nitrous acid produced cross-linking between both chains of the molecule. Should such events lead to mutation, they may be expected to yield pure mutant clones. Tessman's (1959) results with phage, which confirmed the single-chain hit hypothesis, might in fact be more consistent with the hypothesis that nitrous acid produces both single-chain hits (deamination) and double-chain hits (cross-linking). About one-third of the clones obtained by Tessman after nitrous acid treatment were pure, and two-thirds were mosaic. Pure clones did not result from concomitant inactivation of the other DNA strand, because their proportion did not decrease with increasing doses of nitrous acid. C. M. Wieder (personal communication) has found comparable results with coliphage $\mathrm{T}_{2}$. Therefore, either the DNA of one-third of the particles is effectively single stranded for information transfer at the locus studied (for which there is no evidence), or both single- and double-strand mutagenic hits occur when treating phage with nitrous acid. 
This work was performed during the tenure of a fellowship from the Rask Ørsted Foundation and was supported by grants from the Carlsberg Foundation and the Rockefeller Foundation. I am most grateful to Professor M. Westergaard for his generous hospitality, encouragement, and for many discussions; to Mrs Joan Kjemtrup for her able technical assistance; and to Mr Arne Holm (Chemical Laboratory, University of Copenhagen) for the preparation of canavanine sulphate.

This is paper II in the series on forward and back mutation in the pyr-3 region of Neurospora.

\section{REFERENCES}

ATwood, K. C. \& Norman, A. (1949). On the interpretation of multi-hit survival curves. Proc. nat. Acad. Sci., Wash. 35, 696.

Barratt, R. W. \& GARNJOBST, L. (1949). Genetics of a colonial microconidiating mutant strain of Neurospora crassa. Genetics, 34, 351.

BEADLE, G. W. \& TATUM, E. L. (1945). Neurospora. II. Methods of producing and detecting mutations concerned with nutritional requirements. Amer. J. Bot. 32, 678.

Geiduscher, E. P. (1961). 'Reversible' DNA. Proc. nat. Acad. Sci., Wash. 47, 950.

GiLes, N. H. (1951). Studies on the mechanism of reversion in biochemical mutants of Neurospora crassa. Cold Spr. Harb. Symp. quant. Biol. 16, 283.

Grigg, G. W. (1952). Back mutation assay method in microorganisms. Nature, Lond. $169,98$.

Kølmark, G. \& WestergaARD, M. (1952). Validity of the Neurospora back-mutation test. Nature, Lond. 169, 626.

LEA, D. E. (1947). Actions of Radiations on Living Cells. Cambridge University Press.

Mrtchell, M. B. \& MrTChell, H. K. (1954). A partial map of linkage group D in Neurospora crassa. Proc. nat. Acad. Sci., Wash. 40, 436.

ReISsig, J. L. (1958). A suppressor of citrulline dependence in Neurospora: a case of single-gene heterosis. Heredity, 12, 397.

Reissig, J. L. (1959). Forward and back mutation at the pyr 3 locus of Neurospora. Heredity, 13, 144.

REISSIG, J. L. (1960). Forward and back mutation in the pyr 3 region of Neurospora. I. Mutations from arginine dependence to prototrophy. Genet. Res., Camb. 1, 356.

Reissig, J. L. (1963). Spectrum of forward mutants in the pyr-3 region of Neurospora. J. gen. Microbiol. 30, 327.

SneEd, M. C. \& Brasted, R. C. (1956). Comprehensive Inorganic Chemistry, 5, 73. Princeton: D. van Nostrand Co., Inc.

SRB, A. M. \& Horowitz, N. H. (1944). The ornithine cycle in Neurospora and its genetic control. J. biol. Chem. 154, 129.

TeOreli, T. \& Stenhagen, E. (1938). Ein Universalpuffer für den pH-Bereich 2, 0-12,0. Biochem. Z. 299, 416.

Tessman, I. (1959). Mutagenesis in phages X174 and T4 and properties of the genetic material. Virology, 9, 375.

VIELmetTer, W. \& WiEDER, C. M. (1959). Mutagene und inaktivierende Wirkung salpetriger Säure auf freie Partikel des Phagen T2. Z. Naturforsch. 14b, 312.

WestergaARD, M. (1960). Chemical mutagenesis as a tool in macromolecular genetics. In Chemische Mutagenese, Erwin-Baur Gedächtnisvorlesungen 1, 1959. Abh. dtsch. Akad. Wiss., Berl. (Klasse für Medizin), 1.

WestergaArd, M. \& Mitchell, H. K. (1947). Neurospora V. A synthetic medium favoring sexual reproduction. Amer. J. Bot. 34, 573.

WrTkrn, E. M. (1959). Post-irradiation metabolism and the timing of ultraviolet-induced mutations in bacteria. Proc. 10th int. Congr. Genet. 1, 280.

ZeTterberg, G. (1960). The mutagenic effect of 8-ethoxycaffeine, caffeine and dimethylsulphate in the Ophiostoma back-mutation test. Hereditas, 46, 279. 